\title{
Performance Comparison of AODV, DSR and ANODR for Grid Placement Model
}

\author{
Syed Basha Shaik \\ Assistant Professor, \\ C.S.E.Dept, MITS, \\ Madanapalle, A.P, India
}

\author{
Prof. S. P. Setty \\ CS \& SE Dept. Andhra University \\ Visakhapatnam, \\ A.P, India
}

\begin{abstract}
A mobile ad hoc network (MANET) is an autonomous collection of mobile devices that communicate over relatively bandwidth constrained wireless links. Since the nodes are mobile, the network topology may change rapidly and unpredictably over time. Two nodes that are out of one another's transmission range need the support of intermediate nodes which relay messages to set up a communication between each other. This resulted in inventing different routing techniques classified as proactive, reactive and hybrid routing protocols. The reactive routing protocols got much significance because of their low storage requirements and high mobility. AODV and DSR are two such protocols which are being mostly used. The ANODR protocol is a relatively new concept which provides route anonymity and location privacy in addition to on- demand routing capability. In this paper we are exposing the performance of these three protocols in Grid node placement model using QualNet Simulator.
\end{abstract}

\section{General Terms}

This paper exposes the performance of AODV, DSR and ANODR while the nodes are placed in a Grid placement model.

\section{Keywords}

MANET, AODV, DSR, ANODR, Grid.

\section{INTRODUCTION}

A mobile ad-hoc network (MANET) is a collection of nodes, which have the possibility to connect on a wireless medium and form an arbitrary and dynamic network with wireless links. That means that links between the nodes can change during time, new nodes can join the network, and other nodes can leave it [Ref 1]. The set of applications for MANETs is diverse, ranging from small, static networks that are constrained by power sources to large-scale, mobile, highly dynamic networks. A MANET is expected to be of larger size than the radio range of the wireless antennas, because of this fact it could be necessary to route the traffic through a multi-hop path to give two nodes the ability to communicate. A key challenge in Ad Hoc network design is to develop a high quality and efficient routing protocol which can be used to communicate using mobile nodes [Ref 2]. Owing to the nature of unfixed topology in Ad Hoc networks, mobile nodes have to find the delivery path dynamically, maintain the integrity and stability of the path during data delivery process, in order to ensure that data packets are transferred to the destination node, completely. The traditional routing mechanisms and protocols of wired network are inapplicable to Ad Hoc network, thus, the need to use a dynamic routing mechanism in Ad Hoc network [Ref 2].

\section{ADHOC ROUTING PROTOCOLS}

Conventional networks typically rely on distance vector or link state algorithms in which periodic advertisements are sent in order to allow all Routers to keep routing tables upto-date [Ref 9]. Using these algorithms which ensure that the route to every host is always known, presents several problems. First, periodically updating the network topology increases bandwidth overhead. Secondly, repeatedly awakening hosts to receive and send information quickly exhaust batteries [Ref 8]. Then, communication systems often cannot respond quickly enough to dynamic changes in network topology. As shown in Fig 1, routing protocols for Ad Hoc network can be classified into three main categories - Proactive, Reactive, and Hybrid routing protocols. Many protocols have been developed under each category [Ref 1 , $2,3]$.

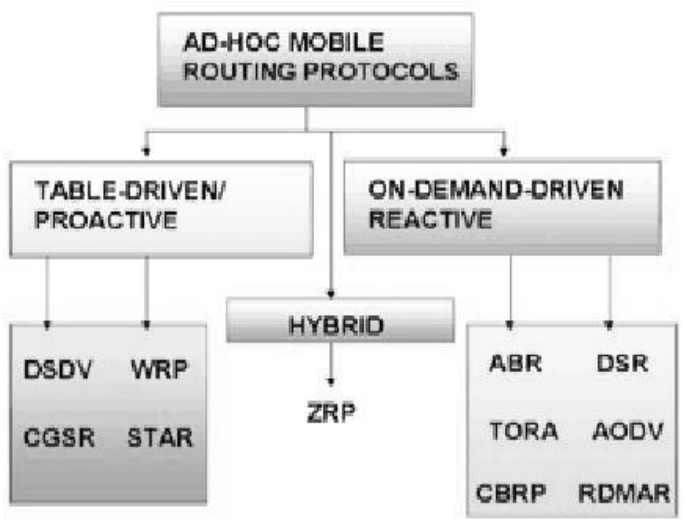

Fig 1. Categorization of Ad Hoc Routing Protocols

In this paper we study three reactive routing protocols namely, Ad Hoc On-Demand Distance Vector (AODV) routing protocol, Dynamic Source Routing (DSR) and Anonymous On-Demand Routing (ANODR) protocol.

\subsection{AODV}

A source node that wants to send a message to a destination for which it does not have a route, broadcasts a request 
RREQ packet. All nodes receiving this packet update their information for the source node and maintain only the next hop's address in a routing table. A RREQ packet contains the source node's address, broadcast ID, current sequence number and the most recent sequence number of the destination node. The response packet RREP is sent by either the destination or a node that has a route to the destination with the sequence number greater than or equal to the sequence number in the RREQ packet. The route is established once the source node receives the RREP. AODV algorithm includes route maintenance facilities. When a link is broken, the related node sends a RERR message to the neighboring nodes using that route. The main advantage of AODV compared to DSR is the reduced bandwidth due to smaller control and data packet. This algorithm has also good scalability because it needs only two addresses: destination and next hop. However, it works with symmetric links and does not allow for multipath routing. So, new routes must be discovered when a link breaks down [Ref 4].

\subsection{DSR}

DSR is a simple algorithm based on the concept of source routing: Source nodes determine routes dynamically and only as needed. A source node that wants to send a packet must check its route cache. If there is a valid entry for the destination, the node sends the packet using that route. If no valid route, the source node initiates the route discovery process: it first send a special route request (RREQ) packet to all neighboring nodes and then propagate through the network collecting the address of all nodes visited until it reaches the destination node or intermediate node with a valid route to the destination node. This node initiates the route reply process: it sends a special route reply RREP packet to the source node providing the sequence of all node through which a packet will travel. This algorithm includes also a route maintenance process. Each host sends a route error (RERR) packet if it encounters a broken link. DSR is easily implemented and thus can work with asymmetric links and involves no overhead when there are no changes in the network. Furthermore, it can be improved to support multiple routes from the source to destination. Nevertheless, large bandwidth overhead is inherent in dynamic source routing. Each route cache collects the addresses of all visited nodes and the RREQ packet can become huge. So, the acceptable network's diameter and its scalability are limited [Ref 5, 7].

\subsection{ANODR}

ANODR address the untraceable routing problem by a route pseudonymity approach [Ref 8]. Each hop on route is associated with a random route pseudonym. Since data forwarding, in the network is based on route pseudonyms, with negligible overhead, local senders and receivers need not reveal their identities in wireless transmission. In other words, the route pseudonymity approach allows to "unlink" (i.e., prevent interference between) network member's location and identity. For each route, they also ensure unlinkability among its route pseudonyms. As a result, in each locality eavesdroppers or any bystander other than the forwarding node can only detect the transmission of wireless packets stamped with random route pseudonyms. It is hard for them to trace how many nodes in the locality, who is the transmitter or receiver, where a packet flow comes from and where it goes to (i.e., what are the previous hops and the next hops on route), let alone the source sender and the destination receiver of the flow. In AODV design a strong adversary with node intrusion capability must carry out a complete "vertex cover" process to trace each on-demand ad hoc route [Ref 6]. The design of route pseudonymity is based on a network security concept called "broadcast with trapdoor information".

\section{GRID PLACEMENT MODEL}

In Grid placement model the mobile nodes are placed as shown in the Fig 2.

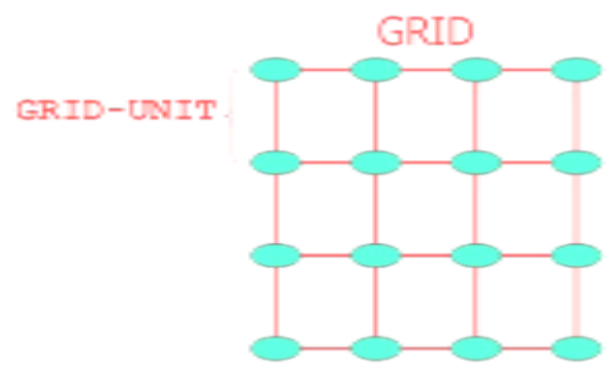

Fig 2. Grid Placement Model

Node placement starts at 0,0 and the nodes are placed in a grid format with each node a GRID-UNIT away from its neighbor [Ref 10]. GRID-UNIT must be specified numerically, with the unit in meters or degrees, depending on the value of COORDINATE-SYSTEM. The number of nodes specified for the simulation must be the square of an integer (for example, 4, 9, 16, 25 ...).

\section{THE TRAFFIC AND MOBILITY MODELS}

Continuous bit rate (CBR) traffic sources are used. The source-destination pairs are spread randomly over the network. The mobility model uses the random waypoint model in a rectangular field with $1500 \mathrm{mX} 1500 \mathrm{~m}$ field whereas network size is varied as $20,40,60,80,100,150$ and 200 nodes. The pause time, which affects the Relative speeds of the mobile hosts, is kept constant at 30s. Simulations are run for 100 simulated seconds. Maximum speed is varied at $0-10 \mathrm{~m} / \mathrm{s}$.

\section{SIMULATION SETUP}

Simulation is done using QualNet5.0.2 simulator. We studied the performance of AODV for three node placement models Grid, Uniform and Random. The performance metrics that we evaluated are average jitter, average end-to-end delay, throughput and packet delivery ratio.

Table 1: Parameter Values

\begin{tabular}{|c|c|}
\hline Parameters & Value \\
\hline Terrain Range & $1500 \mathrm{mX1500m}$ \\
\hline No. of Nodes & $20,40,60,80,100,150$ \\
& and 200 \\
\hline Mobility Model & Random Way Point \\
\hline
\end{tabular}




\begin{tabular}{|c|c|}
\hline & with 30s Pause Time \\
\hline $\begin{array}{c}\text { Simulation } \\
\text { Time }\end{array}$ & 100s \\
\hline
\end{tabular}

\section{RESULTS AND ANALYSIS}

\subsection{Average Jitter}

As can be observed from the Fig 3, the average jitter is very less in AODV at less network size, but increases rapidly with higher network sizes. In DSR it is less at less number of nodes and is more at high number of nodes. But in ANODR the average jitter increases steadily with network size. It has less jitter compared the remaining two. All the results were taken over a terrain of $1500 \mathrm{mX} 1500 \mathrm{~m}$.

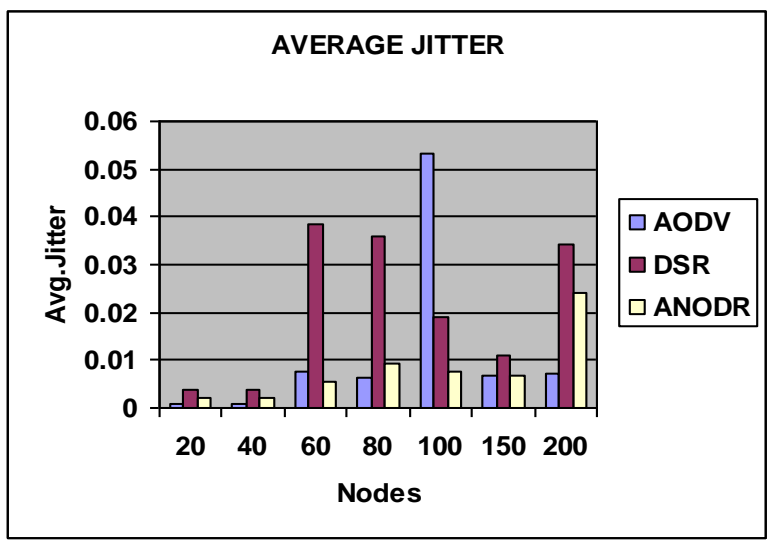

Fig 3. Variation of Average Jitter

\subsection{Average End-to-End Delay}

As can be observed from Fig 4, average end-to-end delay is less in ANODR compared to AODV and DSR. DSR protocol exhibiting highest average end-to-end delay in grid placement and is showing less average end-to-end delay at less network sizes.

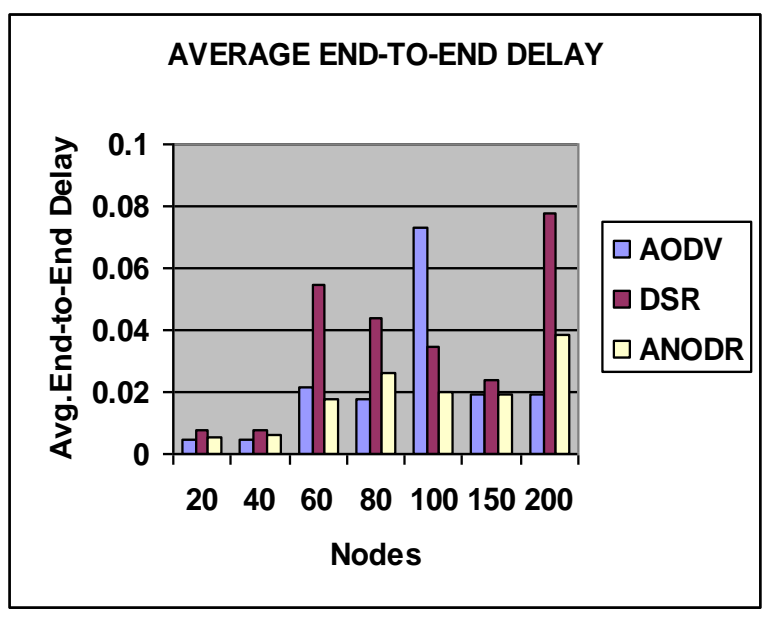

Fig 4. Average End-to-End Delay

\subsection{Throughput}

As can be observed from fig 5, throughput is almost same in all protocols for less network size. But at a network size of 60, 80 and 100 throughput is more in DSR and ANODR, where AODV performing poorly. Again at network sizes of more than 100, AODV and DSR are performing well compared to ANODR.

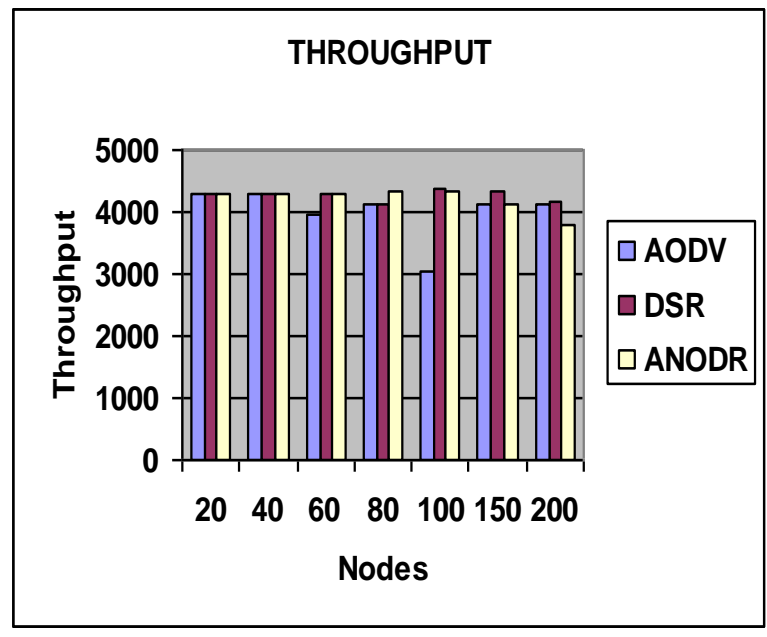

Fig 5. Throughput

\subsection{Packet Delivery ratio}

As can be observed from fig 6, packet delivery ratio is almost same in all AODV, DSR and ANODR protocols at small network sizes. But as the size of network increases, below 100, DSR and ANODR performing well compared to AODV. Further for network size greater then 100, AODV and DSR are performing well compared to ANODR.

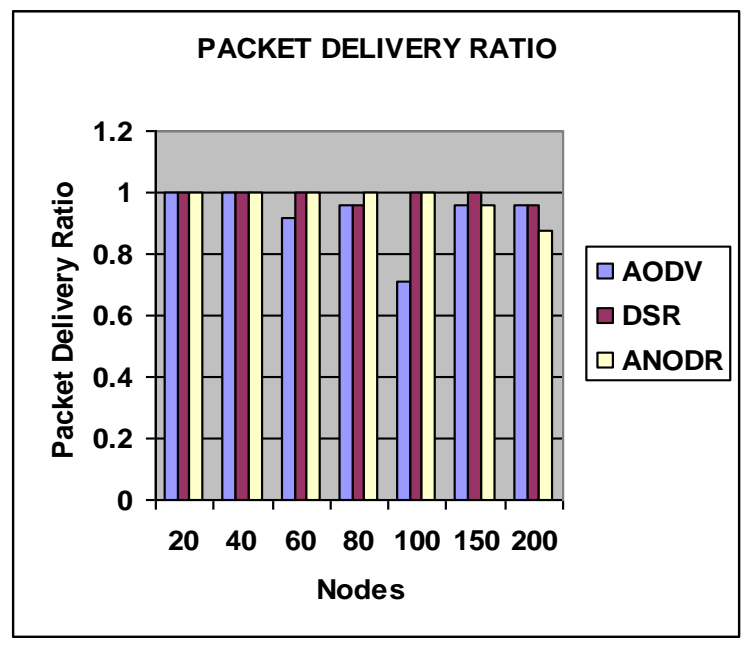

Fig 6. Packet Delivery Ratio

\section{CONCLUSION AND FUTURE WORK}

In our simulation, the performance of AODV, DSR and ANODR in Grid placement model is evaluated for different network sizes, using QualNet5.0.2 simulator. The significance of network size for the performance of AODV, DSR and ANODR protocols is studied. From results we can 
conclude that at less network sizes all the protocols in Grid placement give encouraging results. DSR is giving higher throughput and packet delivery ratio for all network sizes when ANODR giving less average jitter and end-to-end delay.

In future this method of research can be extended to other routing protocols such as TORA, ZRP, LAR1, LMR, ABR, SSI, RDMAR, MSR, AOMDV, MRAODV, and ARA. It can also be extended to proactive routing protocols such as DSDV, WRP etc. Also we extend this research to other placement models such as uniform and random.

\section{REFERENCES}

[1] N Vetrivelan and Dr.A V Reddy (2008). Performance Analysis of Three routing Protocols for Varying MANET Size. Proceedings of the International MultiConference of Engineers and Computer Scientists, Hong Kong.

[2] M.Uma and Dr.G.Padmavathi.A Comparative Study and Performance Evaluation of Reactive Quality of Service Routing Protocols in MANETs. Journal of Theoretical and Applied Information Technology, 2009.

[3] Muhammad Mahmudul Islam, Ronald Pose and Carlo Kopp, (2008). Routing Protocols for Adhoc Networks.

[4] Charles Perkins, Elizabeth Royer, and Samir Das.“Ad hoc on demand distance vector (AODV) routing”. IETF RFC No. 3561, July 2003.
[5] Anuj K. Gupta, Member, IACSIT, Dr. Harsh Sadawarti, Dr. Anil K. Verma. Performance analysis of AODV, DSR \& TORA Routing Protocols. IACSIT International Journal of Engineering and Technology, Vol.2, No.2, April 2010 ISSN: 1793- 8236

[6] Parma Nand, S.C.Sharma \& Rani Astya (2010). Simulation Based Parametric Analysis of AODV Protocol for Adhoc Network. Published in International Journal of Advanced Engineering \& Applications, Jan. 2010.

[7] Nor Surayati Mohamad Usop, Azizol Abdullah,Ahmad Faisal Amri Abidin. Performance Evaluationof AODV, DSDV\&DSR Routing Protocol in Grid Environment. IJCSNS International Journal of Computer Science and Network Security, VOL.9 No.7, July 2009.

[8] Jiejun Kong, Xiaoyan Hong. ANODR: anonymous on demand routing with untraceable routes for mobile adhoc networks. MobiHoc'03, June 1-3, 2003, Annapolis, Maryland, USA

[9] QualNet Network Simulator; Available: http://www.scalable-networks.com.

[10] QualNet documentation, "QualNet 5.0 Model Library: Advanced Wireless"; Available: http://www.scalablenetworks.com/products/qualnet/dow nload.php\#docs 